\title{
Ronsard et la verve licencieuse
}

Sangoul NDONG, Université Assane Seck de Ziguinchor, Sénégal

«Celuy sera véritablement le poète que je cherche en nostre langue, qui me fera indigner, apayser, ejouyr, douloir, aymer, hayr, admirer, etonner, bref, qui tiendra la bride de mes affections, me tournant çà et là à son plaisir. » (Joachim du Bellay 179)

Le Discours des misères de ce temps ${ }^{1}$ reprend le déchirement de la France sous les guerres de Religion. Cela se perçoit dans le ballottement énonciatif de cette œuvre qui répond au tragique de l'époque par le feu de la verve licencieuse dont le trait est l'aspect urgent de la parole engagée, quelque chose du parti pris abrupt et du naturel stylistique où il s'agit pour Pierre de Ronsard d'«écrire pour » (Denis 67) la France et le Catholicisme. La verve licencieuse exprime, dans le Discours des misères de ce temps, une nette intransigeance patriotique. Pour cette raison, elle affecte au langage de Ronsard ce que Roland Barthes appelle une haute fonction transitive (Essais, 152) et traduit l'état d'esprit de Ronsard au lendemain des premières cruautés qui auguraient le déluge de la France. Elle confirme l'autre avis de Barthes suivant lequel « le style est proprement un phénomène d'ordre germinatif qui noue l'humeur de l'écrivain à son langage » (Degré zéro, 16-17). Le ballottement énonciatif, lui, signale la composition mouvementée du Discours des misères de ce temps. Il peut s'expliquer, légitimement, par la diversité des options esthétiques de Ronsard : d'une part, «contois la peine / Et l'extreme malheur dont nostre France est pleine»; de l'autre, «publier, / D’une plume de fer sur un papier d'acier, / Que ses propres enfans l'ont prise et devestue ». Aussi, comme l'éros martial empreint le Discours des misères de ce temps d'accents différents, ce ballottement n'est-il pas sans évoquer l'éclatement des registres que, à propos des Tragiques d'Agrippa d'Aubigné, Jean-Raymond Fanlo appelle « la composition instable » en raison des motifs multiples qui ont inspiré cette œuvre. Dans le cadre de la création militante au seizième siècle, il est question d'une poétique de la fureur qui enchevêtre clichés satiriques et apostrophes ad personae. Ainsi, le Discours ayant appelé contre son auteur la levée des boucliers huguenots ${ }^{2}$, quelles spécificités de la « riposte offensive $\mathrm{e}^{3}$ » du poète vendômois ont-elles inquiété les protestants à l'heure où «l'éloquence du souverain (est) affaiblie, grevée ou altérée à la base » (Poiriet 40) ? Cette inquiétude estelle le signe d'une supériorité de la portée du Discours à celle des libelles huguenots dans les querelles religieuses de la deuxième Renaissance? 
«Le contenu et les enjeux de ce dialogue ont été analysés en profondeur par Jacques Pineaux et Daniel Ménager ; inutile d'y revenir», avance François Rouget dans son «Ronsard et ses adversaires protestants : une relation parodique » (80). Nous revenons sur cette querelle non point pour son contenu et ses enjeux, mais pour mesurer, sur le plan des subtilités énonciatives et argumentatives, le pouvoir de mobilisation du Discours des misères de ce temps et de la Continuation du discours des misères de ce temps contre les protestants. Ce pouvoir de mobilisation désigne la dimension pragmatique du Discours et sa suite, l'efficacité du verbe ronsardien ou les effets escomptés par Ronsard qui, fidèle à son adresse à Guillaume des Autels dans Élégie sur les troubles d'Amboise, veut l'ennemi « en disputant par livres le confondre, / Par livres l'assaillir, par livres luy respondre ». C'est ce que Samuel Junod appelle «la poétique de l'enrollement» (64). La perspective pour appréhender cette portée du dévoilement de « la folie tapie derrière l'apparence de raison » (Sicard) du camp protestant va ici s'orienter dans deux directions. Le repérage du pavé satirique d'abord, ou les misères dont Ronsard fait porter la responsabilité à Théodore de Bèze afin de provoquer chez lui « la répulsion pour la discorde et les troubles de la guerre » (Trotot, «Place des images », 18). Le repérage des jeux d'interpellation ensuite, par lesquels le poète vendômois veut agir sur la conscience de Catherine de Médicis et encore Théodore de Bèze qu'il met en cause pour les exhorter à la Paix. Vu qu'il existe dans le Discours un vacillement entre peinture du tumulte social et interpellations, il semble impératif d'insister sur l'importance de la variation des registres de la censure protestante. Le réquisitoire, qui révolte par le dévoilement de la folie des réformés et de ses dégâts, se disloque dans le Discours et la Continuation au profit d'une dispositio énonciatoire (entendre un ballotement énonciatif) assez fluide où l'imploration s'enchevêtre avec la remontrance. Comment et dans quels buts se marquent ces transitions?

Évaluant « la puissance de l'imprimerie » (Poiriet 43) dans l'espace de l'information et de la rumeur publiques sous l'âge de sang, Ronsard s'oriente vers une poésie d'action avec, pour conséquence, l'« écart de la fable, de la fureur ou d'une langue illustre » (Buron et Gaury 38). Faisant son entrée dans la création militante du seizième siècle, sa conversion poétique à l'épreuve des tueries entre Français met en avant une figure nouvelle du «poète de la chose vue » (Trotot, «Discours de Ronsard», 29) qui le distingue de l'imitateur et du pétrarquiste qu'il fut jusque là. Singulièrement, il étouffe au plus profond de son être la voix de l'inspiration païenne. Tout tient aux appels de l'actualité immédiate, au sens de la responsabilité qui lui intime de rompre toute discordance entre préoccupations sociales en cours et création ${ }^{4}$, ainsi que le précise l'exorde de la Continuation où il clame de vive voix : 
Je serois ou du plomb ou du bois,

Si moy, que la nature a fait naistre François,

Aux siecles advenir je ne contois la peine

Et l'extreme malheur dont nostre France est pleine. (vv. 1-4)

Comme le final de la Continuation où «la Patrie moribonde ou cadavérique » (Junod 61) charge Ronsard de dépeindre son agonie («pren la plume, et d'un stile endurci/ Contre le trait des ans, engrave tout ceci », vv. 441-442), ces vers exhibent l'objet des Discours et insistent sur l'impossibilité d'un choix autre que la dénonciation des cruautés. Ils annoncent, pour Ronsard, un projet scriptural symétrique au passage suivant du Discours des misères de ce temps qui proclame :

Ô toy historien, qui d'ancre non menteuse

Escrits de nostre temps l'histoire monstrueuse,

Raconte à nos enfans tout ce malheur fatal,

Afin qu'en te lisant ils pleurent nostre mal. (vv. 115-118)

Seulement, l'aptitude à «faire naître la pitié ou la colère » (Junod 67) à partir de la représentation du tragique n'est pas la tâche unique du Vendômois. Le mimétisme scriptural est perturbé dans le Discours par le grand usage de l'apostrophe. Porte-parole des nombreuses lamentations de la France affligée, Ronsard prend parti contre les atrocités décrites et jumelle aux violences dénoncées des adjurations poignantes. D'abord, long de quarante-et-un vers, le tableau du tumulte national (vv. 155-196) fléchit l'allégorie de l'Opinion (vv. 127-154) vers la description de la patrie déchirée. La relation du chaos y met en marche une esthétique de l'horreur dans laquelle surgissent les mots de la guerre et de la perversité que sont entre autres : «arme», «sang», «disputent», «desbauche», « couteau », « desreiglé », etc. Mais, à partir du vers 197, la relation de la guerre civile se rompt soudainement avec la conjonction de coordination «mais » pour laisser libre cours à l'imploration suivante où Ronsard supplie directement la Régente de trouver une issue diligente à la crise :

Mais vous, Royne tressage, en voyant ce discord

Pouvez, en commandant, les mettre tous d'accord

[...]

Ainsi presque pour rien la seulle dignité

De vos enfants, de vous, de vostre autorité

(Que pour vostre vertu chaque Etat vous accorde)

Pourra bien appaiser une telle discorde. (Discours, vv. 197-212)

Cette adresse soudaine joue sur l'ethos et le pathos. Elle met doublement l'accent sur ce qui peut donner crédibilité à Catherine de Médicis et honorer ses enfants. Mais comme «l'éloge ronsardien est complexe » (Sicard), juste après l'apostrophe qui précède celle-là de 
façon éloignée dans le Discours (vv. 25-54), retentit aussi aux oreilles de la Régente l'amertume des mânes contre le gâchis du précieux héritage ancestral :

Ha que diront là bas sous les tombes poudreuses

De tant de vaillans Roys les ames genereuses !

Que dira Pharamond! Clodion, et Clovis !

Nos Pepins ! Nos Martels ! nos Charles, nos Loys !

Qui de leur propre sang versé parmy la guerre,

Ont acquis à nos Roys une si belle terre? (Discours, vv. 55-60)

Suffisamment enclins à la pitié envers la France, ces gémissements encore brusques manifestent à l'égard de Catherine de Médicis plus qu'une exhortation au redressement des atrocités. Ils clament l'obligation de pacifier la nation et de perpétuer l'honneur d'antan. Et c'est en cela que les interpellations de conscience de Ronsard sont engageantes. Elles veulent être pragmatiques et faire exécuter à Catherine de Médicis des actions effectives correspondant à leur pouvoir ontologique. Ce pouvoir ontologique est l'instrument propagandiste dont le poète se sert aussi contre les protestants pour les ramener à la raison. En particulier, dans l'époque du seizième siècle, et au-delà, où l'individu est sensible à ce que les contemporains pensent de lui ou que la postérité retiendra, les remontrances à l'égard des réformés via « la voix de la place publique » (Poiriet 46) constituent le reproche tel un moyen de redresser leurs torts. Contre de Bèze, il s'agit d'adjurations incidentes où les accents injonctifs se destinent à l'exposer à la «brimade sociale ${ }^{5}$ ». Pressé d'interpellations sous les yeux de tous, ce chef des protestants de France doit être torturé par les déplorations qui font de lui l'incarnation de l'ingratitude par excellence, dans la négation de tout patriotisme comme c'est le cas ici :

La terre qu'aujourdhuy tu remplis toute d'armes,

$Y$ faisant fourmiller grand nombre de gendarmes,

Et d'avares soldars, qui du pillage ardans,

Naissent desoubs ta voix, tout ainsi des dents

Du grand serpent Thebain les hommes, qui muerent

Le limon en couteaux, dont ils s'entretuerent,

Et nés et demi-nés se firent tous perir,

Si qu'un mesme soleil les vit naistre et mourir,

De Besze, ce n'est pas une terre Gottique,

Ni une region Tartare, ny Scythique,

C'est celle où tu naquis, qui douce te receut,

Alors qu'à Veszelay ta mere te conceut. (Continuation, vv. 99-110)

Sur le plan purement moral, de Bèze et sa suite ont nécessairement quelque chose à se reprocher en lisant ces vers, en mesurant leur responsabilité personnelle dans les tueries entre Français. Avec les entrées inopinées des apostrophes ad hominem, Ronsard verse donc dans la harangue. Ne retenant nullement ce «levain de colère qui enfle sa voix » (Villey 213) 
contre les violences civiles, il vomit une écriture débridée où l'errance discursive traduit l'éclatement de l'instance auctoriale ${ }^{6}$ ainsi que la discordance des registres. Cela implique toutes les formes de dérives rhétoriques qui impriment au Discours son caractère protéiforme vraiment baroque. Cette esthétique baroque se signale par le surgissement, inattendu et péremptoire, des détonations interpellatrices qui entraînent une désagrégation réelle de la composition du Discours, car «Ronsard pratique la rupture de ton, le changement brusque de sujet, la juxtaposition d'éléments qui peuvent paraître hétérogènes » (Buron et Gaury 20). Écrire consiste désormais, pour lui, à prendre en charge les préoccupations sociales de son temps. La façon dont il dévoile la cruauté et interpelle Catherine de Médicis et de Bèze tient à la soumission à l'intransigeance patriotique seule. Nationaliste, son écriture militante fait fi de tout souci de composition régulière. La force des coups de hache de l'adjuration compte, pour lui, plus que l'ordonnance en blocs distincts des clichés du tumulte social et des inflexions indignées que ceux-ci provoquent. N'écrivant que pour l'honneur de la France, Ronsard revêt une psychologie de kamikaze discursif propulsé par les impératifs de son for intérieur. Ses envolées soudaines, les débuts saisissants de ses adjurations farcissent le Discours de transitions discursives imprévues mais accrocheuses et traduisent, du coup, toutes les ressources de l'héritage acquis à l'école de Pindare. Ainsi la création du Discours n'a-t-elle pas de loi, sinon celle qui décrie le tumulte où «la France à jointes mains [...] en prie et reprie » la Reine-mère, où les vers se gorgent d'interpellations poignantes contre les protestants. L'enjeu essentiel de ce style est, bien sûr, l'écoulement de l'intransigeance patriotique, ce qui explique que la verve licencieuse revête quelque chose d'une riposte abrupte et enflammée qui réserve à Ronsard l'attitude de celui qui écrirait sans avoir jamais appris à écrire.

Mais l'idée que le Discours des misères de ce temps est sans art, s'absout avec l'éloquence de la prédication. Car, même si Ronsard semble ne pas se soucier du classement des «accents différents » (Villey 13) de sa verve réprobatrice, même si le zèle religieux n'est pas une qualité de l'inspiration du Discours, sa prédication s'exécute néanmoins avec la plus haute subtilité. Elle exprime un sens, une grande lecture religieuse de la tragédie française. D'abord, à la suite de l'évocation de l'intemporalité des vices (v. 1-20), l'exorde du Discours précise que Dieu se sert des tentations pour éprouver les hommes :

Ainsi il plaist à Dieu de nous exerciter, Et entre bien et mal laisse l'homme habiter, Comme le marinier qui conduit son voyage Ores par le beau temps, et ores par l'orage. (vv. 21-24) 
Très significative, cette précision érige Ronsard en grand prédicateur révélant la volonté de l'Éternel pour prêcher les conditions de la cohésion sociale. Pour lui, toute société qui veut se trouver sur le terrain de la Paix doit, à la différence de Babel, s'abandonner à des mœurs vertueuses. Sa satire, en montrant a posterori les signes des malheurs de l'an soixante deux (vv. 95-114), tire du chaos français une révélation selon laquelle les prétentions imprudentes des réformés sont la «peste » qui a appelé l'ire de

Jupiter faché contre la race

Des hommes, qui vouloient par curieuse audace

Envoyer leurs raisons jusqu'au Ciel pour sçavoir

Les haults secrets que l'homme ne doit voir. (vv. 127-130)

À propos d'affabulation, Claude Blum fait remarquer que « dans la poésie de Ronsard et de la Pléiade, la mythologie permet une reprise sans cesse recommencée, sous mille figures, du récit invariant chrétien » (456). Suivant ce récit, le méchant et le pervers seront toujours punis par là où ils ont péché. C'est cet enseignement christique qui illustre au plus haut degré les misères françaises. Comme les protestants ont brouillé les mœurs de tout le pays, le cataclysme national, tel que l'indique le mythe de l'Opinion, se conçoit telle une malédiction contre les Français «pour n'estre pas sages »(Discours, v. 99). La représentation du chaos où les tabous du sang sont bafoués, donne d'ailleurs à lire l'image de la chute de Babel. Interpellant une deuxième fois la Régente, dont il salue la sagesse, Ronsard la supplie par conséquent de réconcilier tous les Français autour de la Foi, de Bèze et sa suite y compris, en «imitant le pasteur» (v. 199). Tous les registres sont ainsi liés dans le Discours. Ils participent tous d'un art de la persuasion prédicatrice qui d'abord met en évidence l'intemporalité des vices, ensuite indique comment «le comble des pêchés » (Forsyth 263272) appelle le châtiment divin et conduit comme vers un déluge.

Sur un autre angle, la conversation qu'une prédication supposée du chef des protestants de France occasionne entre deux de ses adeptes et Ronsard apparaît comme le vecteur ironique de la réfutation habile de la Réforme. Mettant en évidence le contraste exorbitant entre la mise de de Bèze et l'humilité du Prêtre véritable, Ronsard tourne en dérision son «apparence de raison » (Sicard) en le plaçant devant son imposture :

Un jour en te voyant aller faire ton presche

Ayant de soubs un raistre une espée au costé :

«Mon dieu, ce di-je lors, quelle sainte bonté !

Quelle Evangile helas! quel charitable zelle!» (Continuation, vv. 144147)

Puis, la réplique que le poète apporte au discours de ses deux interlocuteurs. leur expose leurs contradictions et surtout leur désaccord : 
Les apostres jadis preschoient tous d'un accord,

Entre vous aujourdhuy ne regne que discord :

Les uns sont Zvingliens, les autres Lutheristes,

Ecolampadiens, Quintins, Anabaptistes,

Les autres de Calvin vont adorant les pas,

Et l'autre enrage apres l'erreur Muncerienne,

Et bien tost s'ouvrira l'escolle Beszienne. (Continuation, vv. 241-248)

Cette adresse, au même titre que toutes les autres interpellations qui attirent les attentions sur les discordances du Protestantisme, vise à produire chez de Bèze et sa suite encore des effets incommodants. En continuelle dispute avec la tradition et entre eux-mêmes, les réformés sont définitivement dévoyés. Aussi apparaissent-ils dans la Continuation tels de «pauvres incensez » (v. 33). Avec les jeux de contraste et l'ironie, Ronsard réfute habilement ainsi leur religion. La conversation entre deux réformés et lui donne à lire une représentation allégorique des querelles religieuses du seizième siècle, une esthétique de la théâtralisation propice à discréditer le Protestantisme.

Chez Ronsard donc, la verve réprobatrice qui sertit le Discours des misères de ce temps d'une cadence rythmique au plus près de l'oralité enflammée, n'empêche pas la subtilité de l'art de la conversation ${ }^{7}$. Embarqué au sens sartrien du terme, ce farouche ennemi des mœurs travesties épanche par moments un ton d'intransigeance patriotique qui, s'il n'interpelle pas l'honneur des Vallois, empoigne verbalement les protestants. Cela se traduit par des harangues foudroyantes et éclatées qui doivent leur pouvoir de mobilisation non pas seulement à la solennité des supplications, mais à la fermeté des appels à l'ordre également. Mais l'éloquence de la prédication ainsi que les jeux de contrastes piquants permettent aussi à ces harangues d'atteindre les allocutaires de Ronsard dans leur chair.

Pour Christine Pigné, « le conflit avec les protestants a conduit le Vendômois à ramener à une dimension humaine les grandeurs de l'imagination » (2). Le Discours des misères et la Continuation étant dépouillés de furia, d'érudition et de mots recherchés, Ronsard se rend ainsi accessible aux esprits de tous bords. Il est, avec sa poésie antiprotestante, « véritablement le poète » que réclamait du Bellay, celui qui aurait su tenir la bride des affections de ce compagnon si celui-ci fut encore vivant en 1562, à la portée et de plain-pied avec toute la foule des lecteurs du seizième siècle. Et, vu que le Discours et sa suite ont rendu au Catholicisme l'énorme service ${ }^{8}$ que 1'Adversus clandestinos Lutheranos et l'Anti-Calvin ont manqué contre le Protestantisme, vu que «l'échec de la Réforme en France était imputable à Ronsard » (Maurras) et non à Béda ou Pierre Doré, on peut légitimement soutenir qu'à l'épreuve d'une crise sociale, le discours au plus près de l'improvisation se rend 
plus efficient, pragmatiquement parlant, que celui saturé d'érudition dans un style illustré. Car, quand elle «noue l'humeur de l'écrivain à son langage » (Barthes 16-17), la verve de l'orateur indigné, ce presque automatique, se prête à mobiliser plus que tout autre. Elle touche le cœur et l'esprit au plus haut degré.

\section{Bibliographie}

Aristote. La rhétorique, livre II, chapitre I. [œuvre électronique numérisée par Jean-Pierre Murcia]. http://remacle.org

Barthes, Roland. Essais critiques. Paris : Seuil, 1981 (1946).

---. Le degré zéro de l'écriture. Paris : Seuil, 1953.

Bellenger, Yvonne. Introduction. Discours. Derniers vers. Pierre Ronsard. Paris : Garnier Flammarion, 1979. 13-43.

Bergson, Henri. Le rire. Paris : PUF, 1940.

Blum, Claude. La représentation de la mort dans la littérature française de la Renaissance, d'Hélinant de Froidmond à Ronsard, tome 1. Paris : Champion, 1989.

Buron, Emmanuel et Julien Gaury. Lectures de Ronsard. Rennes : PU de Rennes, 2009.

Cotteret, Jean-Marie. La magie du discours. Précis de rhétorique audiovisuelle. Paris: Michalon, 2000.

D’Aubigné, Agrippa. Les Tragiques. Dir. J. Bailbé. Paris : Garnier Flammarion, 1968 (1616).

Denis, Benoît. Littérature et engagement. Paris : Seuil, 2000.

Du Bellay, Joachim. Défense et illustration de la langue française (1549). Paris : Chamard, 1948.

Elliott, Robert C. The Power of Satire : Magic, Ritual, Art. Princeton : Princeton UP, 1960.

Fanlo, Jean-Raymond. Tracés, ruptures. La composition instable des Tragiques. Paris : Champion, 1990.

Forsyth, Elliot. « D’Aubigné, Calvin et "le comble des péchés" ». Mélanges sur la littérature de la Renaissance à la mémoire de Verdum-Louis Saulnier. Genève : n.p. 1984. 263272.

Junod, Samuel. « Comment sortir du cauchemar? Le discours de l'histoire dans les tragédies des guerres de religion ». L'annuaire théâtral : revue québécoise d'études théâtrales, 39 (2006) : 60-74.

Kubera, Jacek. « De l'entre-vie de demi-vivant au sommeil de la paix. Les Derniers vers de Ronsard ». Çédille, Revista de estudios franceses 8 (Avril 2012) : 203-218.

Maurras, Charles. «La politique de Ronsard ». 2008 (1943). 1-11. www.maurras.net 
Ménager, Daniel. Ronsard. Le Roi, le poète et les hommes. Genève : Droz, 1979.

Pigné, Christine. « Le mouvement de l'imagination dans certaines pièces tardives de Ronsard ». Camenae 8 (2010) : 1-19.

Pineaux, Jacques. La polémique protestante contre Ronsard. 2 vols. Paris : STFM / Didier, 1973.

Poiriet, Guy. « L’impuissante éloquence ». Tangences 93 (2010) : 37-49.

Ronsard, Pierre de. Discours. Derniers vers. Pierre de Ronsard. Chronologie, introduction, notes et glossaire Yvonne Bellenger. Paris : Garnier Flammarion, 1979.

---. Continuation du discours des misères de ce temps. 1562. À la Royne. Ronsard. Discours. 81-93.

---. Discours des misères de ce temps. 1562. À la Royne Mere du Roy. Ronsard. Discours.7379.

---. Élégies sur les troubles d'Amboise. 1560. À Guillaume des Autels gentilhomme charrolois. Ronsard. Discours. 53-59.

---. Institution pour l'adolescence du Roy treschrestien Charles Neufviesme de ce nom. 1562. Ronsard. Discours. 67-72.

Rouget, François. «Ronsard et ses adversaires protestants : une relation parodique ». Revue seizième siècle 2 (2006) : 79-94.

Sicard, Claire. « Raison et folie dans les Discours de Ronsard ». En ligne. 4 janvier 2014. http://wp.me/

---. « Ordre et désordre dans les Discours de Ronsard ». En ligne. 4 février 2014. http://wp.me/

Trotot, Caroline. «Les Discours de Ronsard, refus de la rupture historique et invention d'un genre ». Fiction de l'histoire : formes et imaginaires de la rupture. Dir. Zbigniew Przychodniak et Piotr Sniedziewski. Poznan : Société des Amis des Sciences et des Lettres de Poznan, 2012. 19-33.

---. « La place des images dans la poétique ronsardienne de l'histoire ». Histoire et Fiction. Dir. Gisèle Seginger et Piotr Sniedziewski. Poznan : Société des amis des Lettres de Poznan, 2009. 9-24.

Villey, Pierre. Pierre de Ronsard. Textes choisis et commentés. Paris : Plon, 1914.

\section{NOTES}

${ }^{1}$ Nous citons le Discours des misères de ce temps dans la numérotation des vers de l'édition suivante : Ronsard, Pierre de, Discours. Derniers vers, chronologie, introduction, notes et glossaire par Yvonne Bellenger, Paris, Flammarion, 1979. 
${ }^{2}$ On le sait, les Discours du défenseur de la France et du Catholicisme tous en péril «provoquèrent de la part des protestants des répliques indignées» (Villey 205). Bellenger enchérit qu' en effet, les huguenots se déchaînent de 1562 à 1564 dans une série de libelles ». (23). Citons quelques titres recensés par Jacques Pineaux : Palinodies de Pierre de Ronsard sur les Discours des misères de ce temps (de Zamariel); Response aux calomnies contenues au Discours et suyte du Discours sur les misères de ce temps faits par Messire Pierre de Ronsard, jadis Poêle et maintenant Prebstre (de Mont-Dieu); Seconde Response de F. de la Baronie à Messire Pierre de Ronsard prcstre, gentilhomme Vendomois, evesque futur; le Temple de Ronsard ou la légende de sa vie est briesvement descrite; etc.

${ }^{3}$ Cette expression de Charles Maurras (3) traduit la nouvelle forme de lutte que Ronsard jugeait plus appropriée que les gros in-folio latins (tel l'Adversus clandestinos Lutheranos de Béda) et qu'il exprime dans Élégies sur les troubles d'Amboise (1560). À Guillaume des Autels gentilhomme charrolois, vv. 19-22 (53).

${ }^{4}$ Ainsi que le précisent Emmanuel Buron et Julien Gaury, « l'orateur prend à bras le corps les enjeux politiques du moment, et ne les met à distance comme poète. ». Nombreux sont les contemporains de Ronsard qui trempent, eux aussi, leur plume dans le fiel de la verve militante quand l'optimisme humaniste se trouve compromis par ce que Jacek Kubera appelle «le réalisme des luttes armées entre les catholiques et les protestants » (204). Sur cette liste, figure du côté catholique Des Autels. À la France dont maintenant il épouse les intérêts, ce poète adresse dans ses Remontrances : «Mais puisque maintenant pour ton seul bien je veille : / Et que ton seul devoir, France, je te conseille : / Je te pry, mon pays, de ne me debouter, / Mais, ô mon cher pays, je te pry m'escouter »(cité dans Buron et Julien Gaury 37). Du côté protestant, Agrippa d'Aubigné désavoue son pétrarquisme et avertit dès le premier chant de ses Tragiques : «Je n'escris plus les feux d'un amour inconnu, / Mais, par l'affliction plus sage devenu, / J'entreprens bien plus haut, car j'apprens à ma plume / Un autre feu, auquel la France se consume» (I, 55-58). Ces conversions poétiques traduisent par ailleurs une intertextualité conflictuelle fortement prononcée, car les poètes travaillent désormais sur le même matériau historique (les guerres de religions), mais avec des prises de positions radicalement hostiles. Et il semble imprudent de situer Montaigne parmi cette intertextualité, lui qui, le seul presque, se positionne audessus de la mêlée de la guerre des plumes. Mais ce, sans avoir produit avant Les Essais un écrit public attestant un revirement esthétique quelconque de sa part.

${ }^{5}$ L'expression est d'Henri Bergson (103). Robert C. Elliott, lui, considère que « dans toute société où l'on attache une grandeur à l'opinion d'autrui, le ridicule aura un puissant effet dissuasif sur les comportements déviants : plus on redoute la honte, plus on évitera des situations qui pourraient entraîner le discrédit provoqué par la moquerie publique » (60).

${ }^{6}$ Dans le Discours, Ronsard est tantôt poète historien, tantôt poète prédicateur, tantôt poète harangueur.

${ }^{7}$ Celui-ci concerne les techniques de la praxis discursive et leur portée. Pour Aristote, l'objet de la rhétorique étant d'agir à la fois sur le rationnel et l'émotionnel, l'orateur doit s'arroger trois qualités essentielles pour être crédible : la prudence, la vertu et la bienveillance, cf. La rhétorique, livre II, chapitre I. Ces trois qualités se retrouvent chez Ronsard, qui mise aussi sur ce que Jean-Marie Cotteret appelle les trois « $\mathrm{S} »$ de la crédibilité, le « caractère Sensé, Sincère et Sympathique »(35). Ce constat est d'autant plus fondé que Ronsard suit, délibérément, la pente de sa fermeté patriotique en manifestant beaucoup de solidarité avec la France. Non seulement ses apostrophes sont essentielles au redressement des perversités, mais aussi son portrait moral lui assure son audience auprès des allocutaires.

${ }^{8}$ Cf. Villey (205). Maurras conforte cet avis à la page 5 de son même article qui renseigne qu'avec l'entrée de Ronsard dans la bagarre des plumes sous les guerres de religions, «l'espoir changea de camp, le combat changea d'âme ; les catholiques intimidés reprirent confiance en soi. » 